\title{
On the Predictability of Domain-Independent Temporal Planners
}

\author{
ISABEL CENAMOR
}

Computer Science Department, Universidad Carlos III de Madrid, Spain

\author{
MaURo VALLATI
}

School of Computing and Engineering, University of Huddersfield, UK

\section{LUKÁŠ CHRPA}

Department of Computer Science, Czech Technical University, Czech Republic \& Department of Theoretical Computer Science and Mathematical Logic,

\author{
Charles University in Prague, Czech Republic
}

Temporal planning is a research discipline that addresses the problem of generating a totally- or partiallyordered sequence of actions that transform the environment from some initial state to a desired goal state, while taking into account time constraints and actions' duration. For its ability to describe and address temporal constraints, temporal planning is of a critical importance for a wide range of real-world applications. Predicting the performance of temporal planners can lead to significant improvements in the area, as planners can then be combined in order to boost the performance on a given set of problem instances.

This paper investigates the predictability of the state-of-the-art temporal planners by introducing a new set of temporal-specific features, and exploiting them for generating classification and regression Empirical Performance Models (EPMs) of considered planners. EPMs are also tested with regards to their ability to select the most promising planner for efficiently solving a given temporal planning problem.

Our extensive empirical analysis indicates that the introduced set of features allows to generate EPMs that can effectively perform algorithm selection, and the use of EPMs is therefore a promising direction for improving the state-of-the-art of temporal planning, hence fostering the use of planning in real-world applications.

Key words: Automated Planning; Temporal Planning; Predicting Performance 


\section{INTRODUCTION}

Predicting performance of solvers is an important research direction boosting performance via per-instance solver selection as well as providing interesting insights into aspects that affect solvers' behaviour. Prominent examples of successful application of performance predicting techniques can be found in combinatorial search (Kotthoff, 2014), especially in SAT (Xu et al., 2008), ASP (Gebser et al., 2011a), Classical Planning (Fawcett et al., 2014) and Abstract Argumentation (Cerutti et al., 2014).

Predictions are possible by exploiting Empirical Performance Models (EPMs) (Hutter et al., 2014a) which are built by: (i) observing performance of solvers on a large set of training instances; (ii) extracting instance-specific features from each training problem; (iii) learning a predictive model that maps features' value with observed performance. Each feature is either a number or a categorical value that represents a property of the domain or problem model (e.g., the number of objects). Predictions can then be exploited for selecting promising algorithms, or for combining algorithms into a portfolio (Rice, 1976).

EPMs are well established in AI, and have been considered in planning literature since 1990s. Fink (1998) exploited problems size feature for predicting runtime through linear regression, Howe et al. (1999) used five features for predicting the performance of six planners, the subsequent work by Roberts et al. (2008); Roberts and Howe (2009) provided a larger set of features focused on problem models written in PDDL - statistics, and increased the number of considered planners. Most 
recently, Cenamor et al. $(2012,2013)$ further expanded the feature set by including information about the causal and domain transition graphs (Helmert, 2006). Fawcett et al. (2014) considered also features computed by encoding the planning problem as a SAT formula, and by analysing the search space topology. On slightly different tasks, Gerevini et al. (2011) exploited planning features for predicting the length of a makespan-optimal solution plan of a given problem, while Vallati et al. (2015) provided a features-based approach for improving the efficiency of case-base planning systems. State-of-the-art planning EPMs are focused on classical planning, where actions are executed instantly and no numerical or temporal aspects are considered, and they do not guarantee the ability to predict planners performance on more expressive planning models. Real world planning applications, however, usually require to reason also in terms of time constraints; actions are not executed instantly and it might be necessary to run some actions concurrently. Hence, improvements in temporal planning can have a significant impact on most of the planning applications and foster the use of planning in real-world scenario.

In this paper, we:

- introduce a new set of features which are specific to problems dealing with durative actions and temporal constraints,

- combine the introduced features with existing "classical" (propositional) features (Cenamor et al., 2016),

- use the features to generate classification EPMs that predict whether a planner solve a given problem or not, 
- use the features to generate regression EPMs that predict runtime of a planer on a given problem,

- extract a small subset of representative set of features,

- exploit the EPMs for algorithm selection, i.e., selecting the appropriate planning engine for a given problem.

Our extensive empirical analysis aims at demonstrating that i) the generated EPMs are accurate, ii) the selected subset of features is representative, and iii) the algorithm selection method based on the generated EPMs outperforms basic planning engines. Our analysis also provides insights on the state-of-the-art of temporal planning systems that could be fruitfully exploited for improving future planning engines.

The remainder of this paper is organised as follows. Firstly, we discuss related work. We then provide the relevant background on automated planning. Section 4 introduces the set of exploited features. After that, we describe the experimental settings and the framework exploited for the analysis. Then, we analyse the performance of EPMs based on classification and regression and how the EPMs can be exploited for algorithm selection. Finally, we give conclusions.

\section{RELATED WORK}

EPMs can be used to predict the performance of algorithms on previously unseen inputs such as problem instances or parameters settings. One of the early applications of EPMs was in SAT, where EPMs have been used for predicting how much time a 
given algorithm will need to find a solution to a given formula (Hutter et al., 2007, 2014b).

Gomes and Selman (2001) conducted a theoretical and experimental study on the parallel run of stochastic algorithms for solving computationally hard search problems. Their work shows under what conditions running different stochastic algorithms in parallel can give a computational gain over running multiple copies of the same stochastic algorithm in parallel. The empirical hardness of combinatorial problems, which refers to how difficult is to solve a given problem for a given algorithm, has then been studied by Leyton-Brown et al. (2003). More recently, LeytonBrown et al.'s work was extended to create models that are able to predict the runtime of algorithms solving uniform random 3-SAT problems, and the resulting framework was called SATzilla (Xu et al., 2008). SATzilla, which has then been extended for dealing with many different SAT problems, is one of the most successful portfolios at the state of the art, and it has been awarded in many tracks and editions of the SAT competition. ${ }^{1}$ By extracting information from SAT instances, under the form of features, it predicts the runtime of algorithms by using EPMs; on the basis of such predictions, SATzilla selects the most promising solvers to be executed on the given SAT instance.

Another successful portfolio-based approach for SAT is ISAC (Malitsky, 2014), which exploits a pool of different configurations of the same solver. Given a previously unseen instance, ISAC exploits EPMs for selecting the most suitable configuration, in order to minimise the expected runtime.

\footnotetext{
${ }^{1}$ http://www.satcompetition.org
} 
Another area in which EPMs and portfolios approaches has been extensively studied is Answer Set Programming (ASP). A prominent example is Claspfolio (Gebser et al., 2011b), which exploits regression-based EPMs for selecting, among a range of predefined configurations of the well-known ASP solver Clasp (Gebser et al., 2007), the best configuration to minimise the runtime on a given ASP instance. Predictions are made according to a set of features that are extracted from the considered ASP problem. An improved version of Claspfolio, called Claspfolio 2 (Hoos et al., 2014), provides a modular architecture that extends the provided set of techniques by integrating new approaches for extracting features, predicting solvers' performance and combining solvers into a portfolio.

Portfolio approaches have been studied and exploited also in classical planning. BUS (Howe et al., 1999) is the first approach in which a static portfolio has been tested and implemented for solving planning problems. The authors tested the performance of six planners on over 200 problems (all the available benchmarks at that time). According to the observed performance, they then identified a suitable control strategy for combining weaknesses and strengths of the considered planners. Other well-known examples of static portfolios for classical planning include $\mathrm{PbP}$ (Gerevini et al., 2014), Fast Downward Stone Soup (Helmert et al., 2011), and Cedalion (Seipp et al., 2015). These approaches, after observing the performance of a set of planners on training instances, generate a single portfolio that is then used for solving any (previously unseen) planning problem.

EPMs in classical planning have been exploited also for dynamic planning portfolios that combine most promising planners into portfolios according to a given planning instance. IBaCoP2 (Cenamor et al., 2014), which is a good example of a 
dynamic planning portfolio approach, exploits EPMs for selecting the most promising planners (from a given set) for maximising the quality of the solution plans. IBaCoP2 took part in the 2014 edition of the International Planning Competition (IPC), and won the sequential satisficing track (Vallati et al., 2015). Another dynamic portfolio approach, AllPaca (Malitsky et al., 2014), took part in the optimal track of the same competition. AllPaca is a portfolio that selects the most promising optimal planner to run on a given planning task. A comparison of static and dynamic portfolio techniques, focused on optimal planning, has been recently done by Rizzini et al. (2017).

\section{AUTOMATED PLANNING}

Automated planning deals with finding a (partially or totally ordered) sequence of actions transforming the environment from a given initial state to a desired goal state (Ghallab et al., 2004).

\subsection{Classical Planning}

Classical planning assumes a static, deterministic and fully observable environment where action effects are instantaneous.

In the classical representation, the environment is specified via first-order logic predicates. States of the environment are represented as sets atoms, fully grounded predicates. A planning operator $o=\left(\operatorname{name}(o), \operatorname{pre}(o), \operatorname{eff}^{-}(o), \operatorname{eff}^{+}(o)\right)$ is specified such that name $(o)=$ op_name $\left(x_{1}, \ldots, x_{k}\right)$ (op_name is a unique operator name and $x_{1}, \ldots x_{k}$ are variable symbols (arguments) appearing in the operator), $\operatorname{pre}(o)$ is a set of predicates representing the operator's preconditions, $\operatorname{eff}^{-}(o)$ and $\operatorname{eff}^{+}(o)$ are 
sets of predicates representing the operator's negative and positive effects. Actions are fully grounded instances of planning operators. An action $a=\left(\operatorname{pre}(a), \operatorname{eff}^{-}(a)\right.$, $\left.\operatorname{eff}^{+}(a)\right)$ is applicable in a state $s$ if and only if pre $(a) \subseteq s$. Application of $a$ in $s$ (if possible) results in a state $\left(s \backslash \operatorname{eff}^{-}(a)\right) \cup \operatorname{eff}^{+}(a)$.

A planning domain is specified via sets of predicates and planning operators. A planning problem is specified via a planning domain, initial state and set of goal atoms. A solution plan is a sequence of actions such that a consecutive application of the actions in the plan (starting in the initial state) results in a state that satisfies the goal.

\subsection{Temporal Planning}

[Figure 1 about here.]

Temporal planning extends classical planning by incorporating the notion of time. Action application (or execution) takes time and thus action effects might not be instantaneous. In this paper, we consider the restricted form of temporal planning supported in PDDL 2.1 (Fox and Long, 2003) since it is supported by a range of planning engines. Alternatively, temporal planning tasks can be modelled, for instance, in NDDL (Bedrax-Weiss et al., 2005) and solved by using the EUROPA framework (Frank and Jónsson, 2003).

A durative planning operator $o=\left(\operatorname{name}(o), \operatorname{dur}(o), \operatorname{pre}_{S}(o), \operatorname{pre}_{E}(o), \operatorname{pre}_{A}(o)\right.$, $\left.\operatorname{eff}_{S}^{-}(o), \operatorname{eff}_{S}^{+}(o), \operatorname{eff}_{E}^{-}(o), \operatorname{eff}_{E}^{+}(o)\right)$ is specified such that name $(o)=\operatorname{op} \operatorname{id}\left(x_{1}, \ldots, x_{k}\right)$ (op_id is a unique operator name and $x_{1}, \ldots x_{k}$ are variable symbols (arguments) appearing in the operator), $\operatorname{dur}(o)$ represent duration of $o$ 's application, $\operatorname{pre}_{S}(o)$, $\operatorname{pre}_{E}(o), \operatorname{pre}_{A}(o)$ are sets of predicates representing "at start", "at end" and "over all" 
conditions respectively, and $\operatorname{eff}_{S}^{-}(o), \operatorname{eff}_{S}^{+}(o), \operatorname{eff}_{E}^{-}(o), \operatorname{eff}_{E}^{+}(o)$ are sets of predicates representing "at start" negative and positive effects and "at end" negative and positive effects respectively. Durative actions are fully grounded instances of durative planning operators. A durative action $a$ is applicable in a state $s$ and time $t$ if and only if $\operatorname{pre}_{S}(a) \in s$ in $t, \operatorname{pre}_{E}(a) \in s$ in $t+\operatorname{dur}(a)$ and $\operatorname{pre}_{A}(a) \in s$ in $[t, t+\operatorname{dur}(a)]$. The result of application (or execution) of $a$ in $s$ and $t$ (if possible) is such that $\operatorname{eff}_{S}^{-}(a)$ becomes false in $s$ and $t, \operatorname{eff}_{S}^{+}(a)$ becomes true in $s$ and $t, \operatorname{eff}_{E}^{-}(a)$ becomes false in $s$ and $t+\operatorname{dur}(a)$ and $\operatorname{eff}_{E}^{+}(a)$ becomes true in $s$ and $t+\operatorname{dur}(a)$.

Solution plan is a list of pairs $\langle$ action,time $\rangle$ such that each (durative) action is applicable in a current state (starting in the initial state) at time and the result of application of all the actions is a state satisfying the goal.

An example of a temporal operator from the Driver-Log domain is provided in Figure 1. The operator (LOAD-TRUCK) represents loading of an object ?obj into a truck ?truck at a location ?loc.

\section{PROBLEM CHARACTERISATION}

Each planners' performance is predicted by using planning features, which are extracted from the domain and problem specifications. In a nutshell, a feature is a numerical value (either integer or real) that summarises a specific property of a considered specification. A vector of planning features, which provides a succinct yet informative description of a problem instance, is provided to a predictive model. The predictive model, which is learnt accordingly to the observed performance of the given planner on a training set of problem instances, maintains information about 
what features are beneficial or detrimental for the given planner and thus is able to predict its runtime on a previously unseen problem instance.

In this work, we build on existing features introduced for classical planning, and we introduce 71 new features that are specific for temporal planning problems. In total, 139 features are extracted for each problem. The following types of features are extracted:

- PDDL features that are extracted directly from a PDDL domain and problem specification

- SAS+ features (Bäckström and Nebel, 1995) that are extracted from a SAS+ translation of a PDDL domain and problem specification provided by Fast Downward, and its temporal version Temporal Fast Downward (TFD) (Eyerich et al., 2012).

- SAT features that are extracted by ITSAT (Rankooh et al., 2012), which translates a PDDL domain and problem specification into a single SAT formula.

Other approaches such as Torchlight (Hoffmann, 2011) could be a valuable source of features. However, they do not support models that include temporal reasoning, and cannot be exploited in this work.

The considered types of features divided into propositional and temporal are described in detail in the following subsections.

\subsection{Propositional PDDL}

We consider 8 features, listed in Table 1, that are extracted by considering both domain and problem specifications in PDDL. They are a subset of features proposed by Roberts et al. (2008), namely: number of PDDL requirements, number of types, 
objects, predicates, facts in the initial state, number of (non-durative) actions and axioms. Such features can be extracted from classical planning problems and thus are not temporal specific.

[Table 1 about here.]

\subsection{Temporal PDDL}

This class of features, listed in Table 2, considers PDDL elements that appear in temporal models only. For instance, we consider the presence of numeric fluents representing duration of actions, the minimum, maximum, average and the standard deviation of arity of these fluents, the number of conditions and effects that should be fulfilled at the start, in the end or during actions execution (at_start, at_end and over_all). By considering the temporal aspects of PDDL models, it can be derived, for example, if some actions have be run in parallel (one actions achieves an effect at start of its execution and removes it after its execution finishes while another action requires that "effect" during its execution). In total, we consider 31 features in this class.

[Table 2 about here.]

Considering the example operator provided in Figure 1, it can be seen, for example, that it has one at_start effect, one over_all condition.

\subsection{General SAS+}

Many state-of-the-art domain-independent planners exploit SAS+ representation (Bäckström and Nebel, 1995), which can be obtained from PDDL models by the 
Fast Downward framework (Helmert, 2006). Hence, we considered features that can be derived from the SAS+ encoding, which, contrary to predicate-centric PDDL, is object-centric.

The object-centric property of the SAS+ encoding can be exploited to derive Causal Graph (CG) and Domain Transition Graph (DTG). CG encodes information about dependencies between values of state variables, while DTG - generated for each variable - encodes how actions can affect the value of the specific variable. In total, 49 features belong to this class. The non-temporal SAS+ features have already been investigated by Cenamor et al. $(2012,2013)$, and are considered by IBaCoP2 (Cenamor et al., 2014, 2016). Fawcett et al. (2014) also considered a subset of these features in their investigation.

Table 3 shows the list of features extracted from the CG of a problem instance. Table 4 provides the list of the features extracted From the DTGs.

[Table 3 about here.]

[Table 4 about here.]

\subsection{Temporal SAS+}

The SAS+ formalism, originally designed for encoding classical planning problems, has been recently extended for temporal problems (Eyerich et al., 2012). The main difference is in domain transition graphs - called temporal domain transition graphs - that store information about temporal conditions and effects. As previously introduced, in temporal planning problems, conditions can be required to be satisfied at_start, overall or at_end of action execution. In total, 30 features are extracted from 
the temporal SAS+ encoding obtained by Temporal Fast Downward (Eyerich et al., 2012). The features are listed in Tables 5 and 6. Several features are "auxiliar" variables, which Temporal Fast Downward needs for pre-processing purposes: it uses multi-valued state variables and handles logical dependencies and arithmetic subterms via axioms.

[Table 5 about here.]

[Table 6 about here.]

\subsection{SAT Size}

This class of features contains information about the size of a problem encoded in SAT. The only SAT-based solver which is able to handle temporal planning problems is ITSAT (Rankooh et al., 2012). However, for the sake of runtime optimisation, ITSAT (Rankooh et al., 2012), which is so far the only SAT-based solver handling temporal planning problems, generates a file that includes considered SAT variables and some basic relations between them. By using techniques from SATzilla (Xu et al., 2008), we can extract from that file information about the problem size in SAT. In total, 13 features are considered in this class. Details are given in Table 7.

[Table 7 about here.]

\subsection{Feature Extraction}

Feature extraction cutoff time was set to 100 seconds and the RAM has been set to $4 \mathrm{~GB}$. Using too much CPU time for extracting features reduces their usefulness. In the light of the fact that planners tend to solve problems quickly or not at all 
(Howe and Dahlman, 2002), it might be better to select a not-so-good planner than spending too much time to extract all features (and select a better planner).

Table 8 shows the average and maximum time required for extracting the different sets of features as well as the percentage of problems in which the extraction was successfully completed (i.e., within the time and memory bounds). Whereas Propositional PDDL feature extraction requires negligible time, Temporal PDDL feature extraction requires around 10 seconds. On the other hand, extracting SAS+ features is usually more expensive in tens of seconds. SAT size feature extraction, on the other hand, takes about 1-2 seconds. SAS+ features as well as SAT size features have not been computed, due to timeout or running out of memory, in approximately $20 \%$ of the problems considered in our experimental analysis.

[Table 8 about here.]

\section{EXPERIMENTAL SETTINGS}

Our experimental analysis aims at assessing how classification and regression approaches can cope with the problem of algorithm selection for temporal planning problems.

- Classification approaches classify planning problems into a single category, according to the fact whether the planner will solve the problem or not.

- Regression techniques model each planners' runtime.

When dealing with EPMs, a number of decisions have to be taken. Firstly, it is pivotal to select a number of suitable planners; such planners will be used for evaluating the predicting capabilities of classification and regression approaches. 
Secondly, benchmarks have to be gathered for both training and testing purposes. Thirdly, features should be extracted on which EPMs perform predictions. Finally, appropriate metrics have to be considered for measuring the planners' performance. In the next sections, we describe the decisions taken on the mentioned regards. The experimental framework exploited in this analysis is shown in Figure 2. It includes the relevant input and the two main steps, namely training and testing.

[Figure 2 about here.]

Planners and feature extractors were run on a cluster with Intel XEON $2.93 \mathrm{Ghz}$ nodes with 8 GB of RAM each, using Linux Ubuntu 12.04 LTS. Planners had a cutoff time of 1800 seconds and a maximum of 4 GB RAM, while feature extractors has a cutoff time of 100 seconds and a maximum of 4 GB RAM.

\subsection{Planners}

Planning systems that can deal with temporal problems are not as numerous as classical planning solvers. Initially, 12 planners were considered, however, those with very poor performance on training problems (in terms of coverage) were removed. Models for planners with poor coverage on training instances result in a trivial "always negative" EPM, which does always predict that the planner will not solve a given problem is usually built (and is accurate). Such an EPM just never considers these planners in the algorithm selection process. Hence, for our experiments we have considered 8 state-of-the-art temporal planners that accommodate various techniques, namely:

- LPG-td (Gerevini et al., 2003) exploits stochastic local search in the space of 
planning graphs, and is able to generate solutions of increasingly good quality. For the sake of this analysis, as we are interested in runtime performance, LPG was stopped after first solution was found, and seed was fixed.

- POPF2 (Coles et al., 2010) a Forward-Chaining Partial Order Planner that exploits forward-chaining search, expanding nodes according to a partial-order rather than the conventional total-order.

- Yahsp2 and Yahsp2-MT (Vidal, 2011) compute look-ahead plans from deleterelaxed plans and use them in the state-space heuristic search.

- Temporal Fast Downward (TFD) (Eyerich et al., 2012) is based on the Fast Downward planning system and uses an adaptation of the context-enhanced additive heuristic to guide the search in the temporal state space induced by the given planning problem.

- ITSAT (Rankooh et al., 2012) translates the problem into a sequence of SAT instances, corresponding to different time horizons considered for solving the problem instance.

- Yahsp3 and Yahsp3-MT (Vidal, 2014) are latest version of the Yahsp planner, which took part into IPC 2014.

Two different versions (four planning engines) of Yahsp have been included, because it performed well in both IPC 2011 and IPC 2014 (Yashp 3-MT won the temporal track of the IPC 2014). Due to the fact that an EPM is built for each planner, in order to predict its performance, we do not expect the selection of four different engines based on the same planner having an impact on the experimental evaluation. Instead, it may shed some light on the progress of the field. 


\subsection{Benchmarking}

We considered temporal planning problems gathered from the temporal tracks of the last editions of the IPC ${ }^{2}$, namely 2002, 2004, 2006, 2008, 2011 and 2014. Problems not solved by at least one planner were not included in the training set. EPMs have been trained on benchmarks from IPCs 2008 and 2011: in total, 25 domain models and 630 problems have been considered as seen in Table 9. In the IPC-2008, there are two domains having ADL features (in blue) and three domains with numeric-fluents (in green). The IPC-2011 does not include any domain with numeric-fluents nor ADLfeatures.

[Table 9 about here.]

For testing purposes, we designed three different testing sets that are described in Table 10 .

- The IPC 2014 testing set, which includes all the benchmarks from the temporal track of IPC 2014.

- The Known testing set, which considers domains that are also included in the training set. Testing problem instances are different from training ones.

- The Unknown testing set, that includes domains that are not present in the training set.

The IPC 2014 set aims at providing a general overview of the performance of the trained models. The other two testing sets have been designed for evaluating the generalisation ability of trained models on either completely new domain models (Unknown), or new problem instances from already seen domain models (Known).

\footnotetext{
${ }^{2}$ http://icaps-conference.org/index.php/main/competitions
} 
Whenever possible, we considered different encodings of the same domain. Specifically, we considered domain models encoded using STRIPS features only, including numerical constraints, and exploiting ADL features.

[Table 10 about here.]

Having specified the training and testing benchmarks, in this analysis we compare the performance of EPMs using:

- A standard 10-fold cross-validation approach on a uniform random permutation of the training instances.

- the three different testing sets: IPC 2014, Unknown, and Known.

\subsection{Groups of Features}

In order to evaluate how different features affect the ability to predict planners' performance, we consider different groups of features. Features have been grouped according to either the encoding they refer to, or their temporal-specificity, and are summarised in Table 11. All indicates the whole set of computed features (139). PDDL refers to the 49 Features including Propositional, Temporal PDDL, and Problem size. SAS+ considers the 90 features that are extracted by considering the SAS+ encoding only. nT (Non-Temporal) 68 features which are typical of classical planning. Features are gathered from Propositional PDDL and General SAS+ sets. The $\mathbf{T}$ (Temporal) set considers the 71 features that are extracted by considering Temporal PDDL and Temporal SAS+ encoding. We also consider the Sel set, that includes a small number of relevant features that have been automatically selected. Feature selection was done by looking at a J48 decision tree (Quinlan, 1993), which 
is built for predicting the solvability of the training instances, by considering planners as an input information. Given the model, we select the features used in nodes placed in the top fifth of the decision tree. They are believed to be important since, according to the J48 algorithm, they provide the best information gain (Quinlan, 1993). This can be seen as a supervised method for feature selection. Considering top nodes avoids potential overfitting, as it may arise in lower-level leaves of the tree that are used for classifying a very few instances. The accuracy of the EPM generated by the $\mathrm{J} 48$ algorithm is good, approximately $91 \%$. Therefore, we believe information extracted from such a model is relevant. The resulting automatically generated set of features, Sel, includes 11 features: 1 from the Propositional PDDL set, 7 from Temporal PDDL, 2 from General SAS+ and 1 from Temporal SAS+. In particular, the selected features are: the number of predicates included in the domain definition (Propositional PDDL); the number of durative actions, the number of actions that use numeric fluents for representing their duration, the average arity of these fluents, the minimum number of conditions that have to hold at_start of action execution, the maximum number of conditions that have to hold during action execution (over_all), and the minimum and maximum number of effects that become true after action execution finishes (at_end) (Temporal PDDL); the maximum number of outgoing edges of the causal graph, maximum number of incoming edges in the domain transition graph (General SAS+) and; the number of translated durative actions (Temporal SAS+). The selection process emphasises the importance of temporal features ( 8 out of 11 features are taken from temporal sets); they tend to appear earlier in the J48 decision tree and are thus deemed as being more informative. On the other hand, this distribution of selected features across 
SAS+ and PDDL sets requires to extract both PDDL and SAS+ sets of features (the latter is more computationaly expensive).

[Table 11 about here.]

\section{EXPERIMENTAL RESULTS}

Firstly, we assessed the performance of various classification and regression models (45 different algorithms in total), using the WEKA tool (Hall et al., 2009). We considered linear regression, neural networks, Gaussian processes, decision trees, regression methods, clustering, support vector machine and rule-based techniques. 6.1. Classification

For exploiting a classification approach, a different predictive model is built per planner. Such a predictive model has to classify the problem instance according to the fact whether the planner will find its solution or not. Rotation Forest (Rodriguez et al., 2006) performed best among considered classification approaches on the training instances, and is exploited hereinafter. Results are presented in terms of accuracy: it is the number of correct predictions made divided by the total number of predictions made, multiplied by 100 to obtain percentage.

Table 12 shows the results of the trained predictive models on training instances. As expected, performance on training instances is good, regardless of the considered set of features. Usually, any set of features achieves an accuracy of approximately $90 \%$. We conjecture that each class includes at least a few informative features, and that some of the included domains have a large number of corresponding problem 
instances. Larger number of problem instances can positively influence the performance of predictive models because, on a limited and generally coherent set of instances from the same domain, a given planner tend to perform uniformly. It is therefore easier, under such circumstances, for a predictive model to predict the planner's behaviour.

[Table 12 about here.]

The two considered classes (solved, unsolved) have been balanced among all the planners on the training instances; the maximum difference is $40-60 \%$. In order to achieve this class balance, we assessed the initial distribution between classes and, in imbalanced cases, randomly over-sampled the minority class. This approach is common-practice in machine learning (He and Garcia, 2009). The exploitation of training sets with very imbalanced classes will lead to the generation of trivial EPMs that classify all the instances as members of the most represented class.

Summarising, the results in Table 12 clearly indicate that on training instances, the EPMs are able to identify relevant features and combine them for predicting solvability of problems.

Table 13 shows the performance of classification EPMs on the considered testing sets. The analysis of the results on the IPC 2014 set provides a number of interesting insights: (i) the PDDL set leads in 5 out of 8 cases to the best prediction results. (ii) using either temporal or non-temporal set of features achieves similar prediction results; (iii) using all the features together, on the other hand, does not guarantee the best performance; (iv) TFD and Yahsp3 behaviours are hard to predict on testing instances, and (v) the set of selected features usually achieves good prediction 
results, particularly considering that only 11 features are considered for a domainindependent prediction. We observed that TFD and Yahsp2/3 show a very different behaviour on training and testing problems, possibly because of new domains and/or significantly larger instances used in the testing set. TFD translates the PDDL planning problem into $\mathrm{SAS}+$, and then solves the $\mathrm{SAS}+$ problem; the translation phase can be slow and, sometimes, requires a huge amount of memory. On large instances, as those used in the IPC 14, it happens that the translation step fails due to lack of available memory (4 GB); this is clearly hard to predict for an EPM that has been trained on smaller instances, where this issue does rarely arise. Both planners have issues in dealing with problems that need to reason with concurrency in order to be solved. In fact, on the benchmarks of IPC 2014, TFD is not able to solve problems from 5 domains, while Yahsp3 is not able to provide any solution for instances from 3 domains.

\section{[Table 13 about here.]}

Considering all the features at the same time is not always the best option. We believe this is mainly because of introduced "noise". Our hypothesis is supported by the results achieved using the 11 selected features: they represent a (hopefully) noisefree set of features, and their exploitation achieves results close to those achieved when using the All set. The considered sets have some overlap, and this partially explains why in some cases they show similar performance.

Table 13 also shows the results achieved by trained EPMs on the Known and Unknown test sets. We observed that on the Known set, performance is usually less accurate than those achieved on the IPC 2014 testing set. We believe this is due to 
the fact that the domain models are encoded using different sets of PDDL features. In many cases, features introduced in domains that are included in the testing set are not supported by planners. Therefore, predictions are less accurate because, even though many features have values that are similar to some instances included in the training set, the final outcome is completely different. This is also reflected in the very different performance of the considered sets of features. The Known set is significantly smaller than the other sets: from this perspective, mistakes have a much larger impact on the overall evaluation.

ITSAT is the only planner that has very predictable performance on the Known testing set. On the contrary, LPG has quite unpredictable performance on the Known set: for instance, the use of SAS+ and nT features sets leads to around 30\% accuracy. This may be due to the intrinsic randomness of the planning approach exploited by LPG: it is based on stochastic local search. On the other hand, EPMs generated for predicting the performance of Yahsp2, Yahsp3, and TFD tend to have similar accuracy on all the considered testing sets.

To investigate how importance of the features varies between training and testing problems, we applied our selection process on the EPMs built by considering only testing instances. Similarly to the selection process done on training problems, 11 features are selected. One of them is exactly the same: the minimum number of effects that become true when action execution finishes (at_end) (PDDL). Other six features selected according to the testing instances are strongly related to those extracted on training problems, as they consider similar aspects of the problem, but from slightly different perspective: maximum arity of numeric fluents (PDDL), minimum number of at_start conditions (PDDL), minimum duration of an ac- 
tion (PDDL), standard deviation of incoming edges of the domain transition graph (SAS+), number of variables (SAS+), and number of relevant actions (SAS+), Finally, the remaining features are completely different from those included for the EPMs built considering training instances. This is the case of: number of PDDL requirements (PDDL), number of facts in the initial state (PDDL), ratio between the weight and the edges in the causal graph (SAS+), and the ratio between edges and variables of domain transition graph (SAS+).

Overall, considering also the results achieved by the EPMs exploiting the Sel set of features, this analysis confirms their informativeness. It also indicates that the technique we designed for selecting informative features is reasonably accurate, in the sense that it selects features that generalise on different benchmarks.

\subsection{Regression}

Regression EPMs predict the runtime a planner needs to solve a given problem instance. Runtimes of considered planners on selected benchmarks vary between 0 to $1800 \mathrm{CPU}$ seconds. Given the large variations in CPU-times, we trained our regression models to predict the log-runtime rather than absolute time: this has demonstrated to be effective in similar circumstances (Hutter et al., 2014a). To predict when a planner will not be able to solve a given problem instance, we assigned a default value of 2000 CPU-time seconds to unsolved instances. In this way, any predicted value between 1800 and 2000 CPU-time seconds will be considered as that the EPM identified that the given instance will not be solved.

Performance is measured in terms of Root Mean Squared Error (RMSE). Experimentally, we observed that the Decision Tables algorithm (Kohavi, 1995) generates 
- on average - the most accurate predictive models, and we will exploit this approach for the remainder of this experimental analysis.

Table 14 shows the results, in terms of RMSE, of the best regression models with 10-fold cross validation on a uniform random permutation of the 630 training instances. Firstly, we noticed that predicting algorithms runtime is challenging, according to the RMSE values. On the other hand, it is well-known that RMSE is sensitive to occasional large errors (e.g., predicting an instance as unsolvable although it can be solved quickly), thus actual predictions can be better, on average.

[Table 14 about here.]

Table 15 shows the RMSE results achieved by the regression predictive models on the three considered testing sets. Differently from the results of classification EPMs, regression models are providing the most accurate predictions on the Known test set. On the other test sets, regression models tend to perform similarly. However, as for the classification models, ITSAT's perfomance is the easiest to predict. On the Known testing set, the RMSE goes below 1 because ITSAT does not solve the vast majority of the problems, and therefore the EPM tends to predict very poor performance.

[Table 15 about here.]

We noticed that the regression approach shows similar RMSE performance for the TFD planner on training and testing instances. This was not the case for the classification model. On the other hand, we observed that Yahsp-based systems show a very different behaviour on the training and testing instances as in the classification case. In particular, the behaviour of the MT versions are the most challenging to 
predict. Since Yahsp-MT exploits a multi-threaded approach, it is possibly more sensitive to small changes of the execution environment (e.g., operative system calls, input/output delays). This has a limited impact on the ability of the planner in solving instances, but makes the actual runtime harder to predict. A similar explanation can be provided for the high error in the LPG predictions: LPG exploits a randomised search algorithm that, in presence of domain models that are similar to those used in training instances, lead the predictive model to make inaccurate estimations.

With regards to the different classes of features, using the Sel set often results in the best regression EPMs since, very likely, this set is noise-free and very informative. We also observed that the features from the temporal set are very informative and achieve prediction performance that is usually very close to the best.

\subsection{Exploiting EPMs for Algorithm Selection}

After evaluating prediction performance of the classification and regression EPMs, we are in position to exploit them for performing on-line algorithm selection. In particular, we tested the capability of EPMs as mechanisms for selecting the most promising planner to exploit on a given (and previously unseen) testing instance. A single planner is selected for solving each planning instance, and a cutoff time of 1800 second is allocated to the selected planner.

Classification EPMs are able to predict whether a given planner will solve a given problem instance, or not. Therefore, they can be used to select planners in order to maximise coverage, i.e. the number of solved instances. As a different classification EPM is generated for each planning engine, the selection is performed as follows. 
Among all the planners that are predicted to solve a given problem, the selected planner corresponds to the EPM that showed the best accuracy on training instances.

Regression EPMs predict, for each planner, the runtime needed to solve a given planning instance. The planner selected is the one predicted to be the fastest.

We compare the approaches by considering the IPC runtime score and the coverage. The IPC score is defined as in the Agile track of the IPC 2014. For a planner $\mathcal{C}$ and a problem $p, \operatorname{Score}(\mathcal{C}, p)$ is 0 if $p$ is unsolved, and $1 /\left(1+\log _{10}\left(T_{p}(\mathcal{C}) / T_{p}^{*}\right)\right)$, where $T_{p}(\mathcal{C})$ is the CPU-time needed by planner $\mathcal{C}$ to solve problem $p$ and $T_{p}^{*}$ is the CPU-time needed by the best considered planner, otherwise. The IPC score on a set of problems is given by the sum of the scores achieved on each considered problem.

In terms of basic planners' performance on the IPC 2014 testing set, Figure 3 shows the corresponding number of solved problems, with regards to CPU time. Most of the planners are usually either solving instances quickly, or not at all. Exceptions are ITSAT and TFD that are able to solve a few instances in about 600 seconds and a few more instances in about 1400 seconds.

[Figure 3 about here.]

Table 16 shows the results, in terms of number of solved problems and IPC runtime score achieved on the IPC 2014 test set by the classification and regressions EPMs using different sets of features. In this analysis we ignore the CPU-time needed for extracting features, as the main goal of this section is to evaluate the ability of the generated EPMs to effectively select a suitable planner for a given problem.

We focus on four groups of features: All, Sel, Temporal, and non-Temporal. 
For algorithm selection, we are particularly interested in assessing the usefulness of temporal-specific features and in evaluating the effectiveness of the small set of selected features.

[Table 16 about here.]

For the sake of comparison, Table 16 includes the performance of the virtual best solver (VBS) which represents an Oracle that selects always the best possible planner for solving the specific problem, the two best basic solvers accordingly to (C)overage (LPG) and IPC (S)core (Yahsp2), and a static portfolio (B4P), which includes the best 4 planners according to coverage performance on testing instances: LPG, Yashp2, Yashp3 and TFD. The solvers are ordered according to their coverage (descending order) and each planner runs for 1/4 of the cutoff time (i.e., 450 seconds per planner). Considering these additional systems - VBS, B4P and the best basic solvers - provides a better and more complete understanding of the performance of algorithm selection across the EPMs.

Both classification and regression EPMs achieve better coverage results than the best basic solver $(+11 \%$ and $+32.5 \%$, respectively). Performance achieved by using the regression EPMs is very close to performance of the VBS and better than the B4P.

It is useful to remind that the $\mathrm{B} 4 \mathrm{P}$ has been configured by considering the performance of planners on testing instances, while both regression and classification EPMs have been trained on a different set of instances. From this perspective, the proposed EPMs demonstrate ability to generalise, since they provide useful prediction for performing algorithm selection on unseen instances, although we observed 
that the regression EPMs outperform the classification EPMs, both in terms of coverage and IPC score. This is due to the fact that the classification EPMs do not estimate the performance difference between solvers, so an error in the prediction might result in selecting a planner that will not solve the problem. The regression EPMs consider planners' runtime. Therefore, a mistakenly selected planner usually needs a longer execution runtime while a planner with extremely poor performance is very rarely selected.

With regards to the considered sets of features, we noticed a very different behaviour of the classification and regression EPMS. Classification achieves the best coverage performance when using the selected set of 11 features; the IPC score on that set is close to the best one, which is achieved by using Temporal features. On the other hand, the Sel set is not the most informative for algorithm selection through regression; using the whole set of features - or even the set including only temporal / non temporal features - achieves better performance.

In Table 16 domains are listed according to the difficulty of their instances. In this context, the smaller is the number of planners that can solve all the problems, the more difficult the domain is. According to this intuitive definition, the less difficult (easier) domain is Parking, since 6 planners solve all the problems, and all the considered planners solve at least 6 instances. The two more difficult domains are TMS, because only one planner is able to solve all its benchmark problems, and TurnAndOpen, where 3 planners solve about 10 problems each. We conjecture that the difficulty of domains play a pivotal role in algorithm selection. If a difficult domain is included in the training set, it is easier for the EPM to correctly identify the planner(s) to exploit on the corresponding testing instances. On the other hand, 
if the domain is not considered in the training set, the capability of the EPMsbased approach of selecting the good planner depends only on the informativeness of features and generalisation.

Figure 4 provides an overview of the empirical difficulty of the testing domains used in the IPC 2014, both from planning and instances perspective. The red line (Solved Problems) represents the proportion of problems solved per domain. A value of 1 indicates that all the planners are able to solve all the testing problems; on the contrary, the value of 0 means that no planner can solve any of the testing problems. Similarly, the green line (Planners) reports the planners' perspective, as the proportion of planners that can solve all the problems of a domain. Figure 4 clearly shows that out of the considered domains, 4 are extremely difficult for the state-of-theart domain-independent planners. The difficulty of TMS and TurnAndOpen derives from the fact that their problems need actions to be executed concurrently in order to be solved.

[Figure 4 about here.]

Table 17 shows the results, in terms of number of solved problems and IPC runtime score of the considered classification and regressions EPMs, using different sets of features, on the Unknown and Known testing sets. On these testing sets, the best basic solver according to either coverage or runtime, is LPG. LPG provides better coverage results than the proposed classification and regression based algorithm selection approaches. This is true also for the B4P static portfolio that, in fact, includes LPG as well. The best basic planner and the static portfolio are selected (configured) according to the performance of considered planners on the testing 
instances, so they are exploiting information that is not available to the algorithm selection approaches and that is not available before having the instances solved. Algorithm selection approaches rely on a single (selected) planner for generating a solution for a given planning problem; instead the B4P can fully exploit the available CPU-time for running 4 planners for a considerable amount of time (LPG, Yashp2, Yashp3 and TFD).

[Table 17 about here.]

Algorithm selection techniques aim to select planners that solve the given problem instances in minimum time. In the case of regression techniques, predictedto-be-fastest planners are selected in order to solve the given problem instance; classification-based selection instead tries to identify a planner that will solve the problem regardless of the expected runtime. However, as observed in our experiments, the classification-based approaches underperform the regression approaches.

On the Unknown testing set, we observed that the algorithm selection approaches are struggling with domains in which very few planners are able to solve some instances. This is the case, for example, of the UMTS domain: the regression approaches tend to select LPG, that is not able to solve any problem. On such a test set, we noticed that using - instead of a portfolio - a single planner that shows the best coverage on training instances does not necessarily lead to the best results.

Analysing of importance of each features' set, we made several observations. The Sel set achieves good performance in the classification approach (see Table 16. The only exception can be observed on the Unknown test set: in that case, despite a remarkably high IPC score, the number of solved instances is significantly lower 
than those achieved when exploiting different sets of features (see Table 17). On the Unknown testing set the use of non-Temporal features leads to the best performance of the classification EPMs approaches (see Table 17). Temporal features, on the other hand, are useful for the regression approaches on the Unknown test sets (see Table 17).

The results shown in Table 17 confirm that the regression EPMs are able to effectively select planners for solving previously unseen instances and show that the very small set of selected features (Sel) is a valuable source of information for performing algorithm selection on either previously seen or previously unseen domains and problem instances.

[Table 18 about here.]

\subsection{Discussion}

The algorithm selection approaches presented in the previous section exploit EPMs for selecting a single planner for solving a given planning problem. In this section, we shed some light on the selected planners.

As Table 16 shows, on the 4 "unseen" domains in the IPC 2014 set (highlighted in grey in the table), the regression approaches tend to provide better prediction performance on average, so they are able to better generalise on previously unseen domains; the classification approaches are unable to select a good planner for the RTAM domain while they are able to identify a suitable planner for the MapAnalyser domain. Table 18 shows the planners selected by the particular EPMs using the different sets of features. The classification approaches usually exploit more different planners per domain. In every domain except Floortile, the regression approaches 
select one single planner per a set of features (in MatchCellar and DriverLog different planners were selected while considering a different set of features). This, in combination with results shown in Table 16, supports the observation that a single planner usually performs well on problems from the same domain. However, we conjecture that this is due to the fact that benchmarks for IPCs are usually selected from a homogeneous distribution and are generated using a single problem generator. This can lead to structurally-similar problem instances, on which a single planner can excel.

By analysing the results shown in Table 18, we can derive that the difference of performance between the regression EPMs using the selected set of features, and the other sets, mainly arises in the Driverlog domain. In that domain, TFD does not solve any problem, thus selecting it has a detrimental effect on performance. The winner of the IPC-14 temporal track - Yahsp3-M T- is never selected by the regression EPMs and is selected only in one domain by the classification EPMs. Similarly, the previous version of that planner is rarely used. This is possibly due to the fact that these planners show impressive performance on a very limited number of domains, particularly RTAM and MapAnalyser, which are not included in the training set. We also noticed the remarkable performance of the LPG planner; even though it has been developed more than a decade ago, it is competitive with the current state-ofthe-art of temporal planning. Finally, Table 19 summarises the number of times that each planner was selected by the considered EPMs.

[Table 19 about here.] 


\section{CONCLUSION}

In this paper, we filled the gap between classical and temporal planning in terms of predicting planners' performance. Our work establishes a new extensive set of features that can be extracted from temporal planning problems. In particular, we introduced 71 new temporal-specific features, and merged them with "classical" (propositional) features that can be extracted also from temporal problems; in total 139 planning-specific features have been considered for generating both classification and regression EPMs which are exploited to select on-line the planner for solving a given planning task. The large empirical analysis performed in this work: (i) demonstrates that the performance of many temporal planners can be accurately predicted by using EPMs; (ii) gives insights into the motivations that make planners hard to predict, particularly running out of memory and the concurrency requirements; (iii) provides a valuable and informative set of 11 features that can be used for effectively predicting the performance of temporal planners; (iv) shows that both temporal-specific and non temporal features are useful for predicting planners performance; (v) demonstrates that using EPMs for algorithm selection can significantly improve the current state-of-the-art of temporal planning. Our work also highlights a worrying evidence: in terms of coverage, planners that have been introduced more than a decade ago are able to achieve performance comparable - and often better with the most recent planning systems. LPG results emphasised this idea, in many cases it works better than the more recent planners.

Future work includes the extension of the current set of features by considering probing features - information gained by short runs of different solvers -, and the 
integration of different planners' configurations obtained by using algorithm configuration tools, such as SMAC Hutter et al. (2011). Finally, we plan to test the suitability of deep learning approaches for generating EPMs.

\section{Acknowledgements}

This research was funded by the Czech Science Foundation (project no. 1807252S) and by the OP VVV funded project CZ.02.1.01/0.0/0.0/16_019/0000765 "Research Center for Informatics". The authors would like to acknowledge the use of the University of Huddersfield Queensgate Grid in carrying out this work.

\section{REFERENCES}

B ÄCKSTRÖM, Christer, and Bernhard NeBel. 1995. Complexity results for SAS+ planning. Computational Intelligence, 11:625-656.

BedraX-Weiss, Tania, Conor McGann, Andrew Bachmann, Will Edgington, and Michael IATAURO. 2005. EUROPA2: User and contributor guide. In NASA Tech. Rep.

Cenamor, Isabel, Tomás de la Rosa, and Fernando Fernández. 2012. Mining IPC-2011 results. In Proceedings of the 3rd workshop on the International Planning Competition.

CEnAmor, Is ABel, Tomás de LA RosA, and FernANdo Fernández. 2013. Learning predictive models to configure planning portfolios. In Proceedings of the 4th workshop on Planning and Learning (ICAPS-PAL 2013), pp. 14-22.

Cenamor, Is ABel, Tomás de la Rosa, and Fernando Fernández. 2014. IBaCoP and IBaCoP2 planner. In Proceedings of the 8th International Planning Competition.

Cenamor, Is ABel, Tomás de la Rosa, and Fernando Fernández. 2016. The IBaCoP planning system: Instance-based configured portfolio. Journal of Artificial Intelligence Research, 56:429-464.

Cerutti, Federico, Massimiliano Giacomin, and Mauro Vallati. 2014. Algorithm selection for preferred extensions enumeration. Proceedings of COMMA, 266:221-232.

Coles, A. J., A. I. Coles, M. Fox, and D. Long. 2010. Forward-chaining partial-order planning. In Pro- 
ceedings of ICAPS.

Eyerich, Patrick, Robert MAtTmüller, and Gabriele Röger. 2012. Using the context-enhanced additive heuristic for temporal and numeric planning. In Towards Service Robots for Everyday Environments. Springer, pp. 49-64.

Fawcett, Chris, Mauro Vallati, Frank Hutter, Jörg Hoffmann, Holger H. Hoos, and Kevin LEYTON-BROWN. 2014. Improved features for runtime prediction of domain-independent planners. In Proceedings of ICAPS, pp. 355-359.

FINK, EUGENE. 1998. How to solve it automatically: Selection among problem-solving methods. In Proceedings of AIPS, pp. 128-136.

Fox, MARIA, and DeREK LONG. 2003. PDDL2.1: An extension to PDDL for expressing temporal planning domains. Journal of Artificial Intelligence Research, 20:61-124.

FrAnK, JEREMY, and ARI K. JónsSON. 2003. Constraint-based attribute and interval planning. Constraints, 8(4):339-364. . https: / / doi .org/10.1023/A:1025842019552.

Gebser, Martin, Roland Kaminski, Benjamin Kaufmann, Torsten Schaub, Marius Thomas Schneider, and Stefan ZiLler. 2011a. A Portfolio Solver for Answer Set Programming: Preliminary Report, pp. 352-357. Berlin, Heidelberg: Springer Berlin Heidelberg. ISBN 978-3-642-20895-9. . http: //dx.doi.org/10.1007/978-3-642-20895-9_40.

Gebser, Martin, Roland Kaminski, Benjamin Kaufmann, Torsten Schaub, Marius Thomas SCHNEIDER, and Stefan ZiLler. 2011b. A portfolio solver for answer set programming: Preliminary report. In Proceedings of the 11th International Conference Logic Programming and Nonmonotonic Reasoning (LPNMR), Springer, pp. 352-357.

Gebser, Martin, Benjamin Kaufmann, André Neumann, and Torsten Schaub. 2007. clasp : A conflict-driven answer set solver. In Proceedings of the 9th International Conference Logic Programming and Nonmonotonic Reasoning (LPNMR), Springer, pp. 260-265.

Gerevini, Alfonso, Alessandro Saetti, and IVan Serina. 2003. Planning through stochastic local search and temporal action graphs in LPG. J. Artif. Intell. Res.(JAIR), 20:239-290.

Gerevini, Alfonso, Alessandro Saetti, and Mauro Vallati. 2011. Exploiting macro-actions and predicting plan length in planning as satisfiability. In Proceedings of AI*IA, pp. 189-200.

Gerevini, Alfonso, Alessandro Saetti, and Mauro Vallati. 2014. Planning through automatic portfolio configuration: The pbp approach. J. Artif. Intell. Res., 50:639-696.

Ghallab, Malik, Dana Nau, and Paolo Traverso. 2004. Automated Planning: Theory \& Practice. 
Morgan Kaufmann Publishers.

Gomes, Carla P., and Bart Selman. 2001. Algorithm portfolios. Artificial Intelligence, 126(1-2):43-62.

Hall, Mark, Eibe Frank, Geoffrey Holmes, Bernhard Pfahringer, Peter Reutemann, and IAN H. WITTEN. 2009. The WEKA data mining software: An update. SIGKDD Explorations, 11(1):10-18.

He, Haibo, and Edwardo A Garcia. 2009. Learning from imbalanced data. IEEE Transactions on Knowledge and Data Engineering, 21(9):1263-1284.

Helmert, MALte. 2006. The Fast Downward planning system. J. Artif. Intell. Res.(JAIR), 26:191-246.

Helmert, Malte, Gabriele RöGer, and Erez Karpas. 2011. Fast downward stone soup: A baseline for building planner portfolios. In ICAPS 2011 Workshop on Planning and Learning, pp. 28-35.

Hoffmann, J. 2011. Analyzing search topology without running any search: On the connection between causal graphs and h+. J. Artif. Intell. Res.(JAIR), 41:155-229.

Hoos, Holger, Marius Thomas Lindauer, and Torsten Schaub. 2014. claspfolio 2: Advances in algorithm selection for answer set programming. Theory and Practice of Logic Programming, 14(4-5):569585.

Howe, Adele, and Eric Dahlman. 2002. A critical assessment of benchmark comparison in planning. J. Artif. Intell. Res.(JAIR), 17:1 - 33.

Howe, Adele, Eric Dahlman, Chistopher Hansen, Anneliese Von Mayrhauser, and Michael SCHEETZ. 1999. Exploiting competitive planner performance. In Proceedings of (ECP), pp. 62-72.

Hutter, F., H. H. Hoos, and K. Leyton-Brown. 2011. Sequential model-based optimization for general algorithm configuration. In Proceedings of LION, pp. 507-523.

Hutter, Frank, Holger H Hoos, and Thomas StÜtZle. 2007. Automatic algorithm configuration based on local search. In AAAI, Volume 7, pp. 1152-1157.

Hutter, Frank, Lin Xu, Holger H Hoos, and Kevin Leyton-Brown. 2014a. Algorithm runtime prediction: Methods \& evaluation. Artificial Intelligence, 206:79-111.

Hutter, Frank, Lin Xu, Holger H Hoos, and Kevin Leyton-Brown. 2014b. Algorithm runtime prediction: Methods \& evaluation. Artificial Intelligence, 206:79-111.

KoHaVi, Ron. 1995. The power of decision tables. In Machine Learning: ECML-95. Springer, pp. 174-189.

KotThoff, LARs. 2014. Algorithm selection for combinatorial search problems: A survey. AI Magazine, 35(3):48-60.

Leyton-Brown, Kevin, Eugene Nudelman, Galen Andrew, Jim McFadden, and Yoav Shoham. 2003. A portfolio approach to algorithm selection. In IJCAI, Volume 1543, p. 2003. 
MALITSKY, YURI. 2014. Instance-specific algorithm configuration. In Instance-Specific Algorithm Configuration. Springer, pp. 15-24.

MALitsky, YURI, DAVID WAng, and EReZ KarPas. 2014. The allpaca planner: All planners automatic choice algorithm. In International Planning Competition (IPC), pp. 71-73.

QUinlan, JoHn Ross. 1993. C4. 5: programs for machine learning, Volume 1. Morgan kaufmann.

Rankooh, Masood Feyzbakhsh, Ali Mahjoob, and Gholamreza Ghassem-Sani. 2012. Using satisfiability for non-optimal temporal planning. In Logics in Artificial Intelligence. Springer, pp. 176188.

Rice, John R. 1976. The algorithm selection problem. Advances in Computers, 15:65 - 118.

Rizzini, Mattia, Chris Fawcett, Mauro Vallati, Alfonso Emilio Gerevini, and Holger H. Hoos. 2017. Static and dynamic portfolio methods for optimal planning: An empirical analysis. International Journal on Artificial Intelligence Tools, 26(1):1-27.

RoBerts, MARK, and Adele Howe. 2009. Learning from planner performance. Artificial Intelligence, 173(56):536-561.

Roberts, Mark, Adele E. Howe, Brandon Wilson, and Marie desJardins. 2008. What makes planners predictable? In Proceedings of ICAPS, pp. 288-295.

Rodriguez, Juan José, Ludmila I Kuncheva, and Carlos J Alonso. 2006. Rotation forest: A new classifier ensemble method. IEEE Transactions on Pattern Analysis and Machine Intelligence, 28(10):16191630.

Seipp, Jendrik, Silvan Sievers, Malte Helmert, and Frank Hutter. 2015. Automatic configuration of sequential planning portfolios. In Proceedings of the Twenty-Ninth AAAI Conference on Artificial Intelligence, pp. 3364-3370.

Vallati, Mauro, Lukáš Chrpa, Marek Grzes, Thomas L McCluskey, Mark Roberts, and Scott SANNER. 2015. The 2014 international planning competition: Progress and trends. AI Magazine, 36(3):9098.

Vallati, Mauro, Ivan Serina, Alessandro Saetti, and Alfonso Emilio Gerevini. 2015. Identifying and exploiting features for effective plan retrieval in case-based planning. In Proceedings of ICAPS, pp. 239-243.

Vidal, Vincent. 2011. YAHSP2: Keep it simple, stupid. In Proceedings of the 7th International Planning Competition, pp. 83-90.

VIDAL, VINCENT. 2014. YAHSP3 and YAHSP3-MT in the 8th international planning competition. In Proceed- 
ings of the 8th International Planning Competition.

Xu, Lin, Frank Hutter, Holger H Hoos, and Kevin Leyton-Brown. 2008. SATzilla: Portfolio-based algorithm selection for SAT. J. Artif. Intell. Res.(JAIR), 32:565-606. 


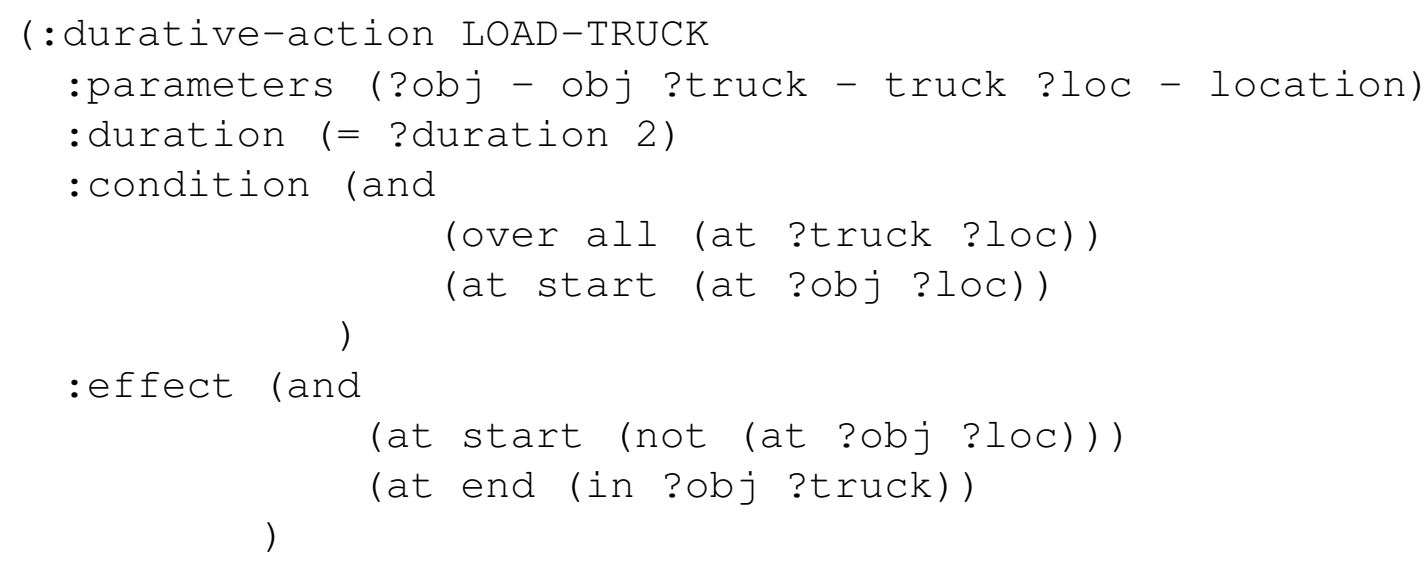

FIGURE 1. An example of a durative operator encoded in PDDL 2.1. 


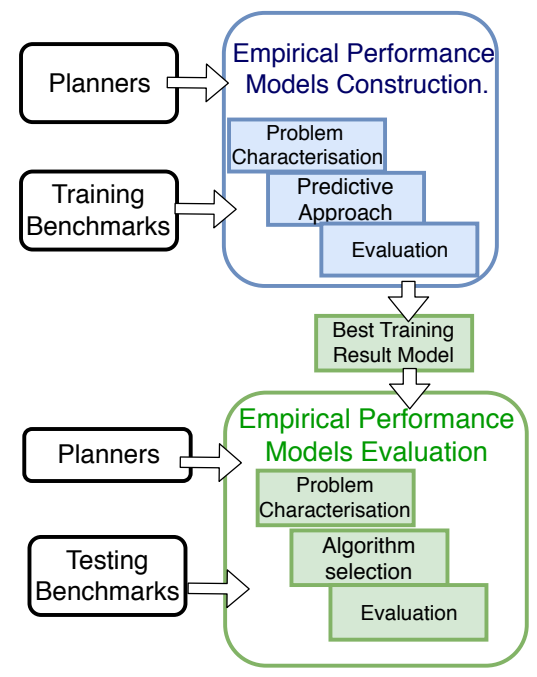

FIGURE 2. The architecture of the proposed system 


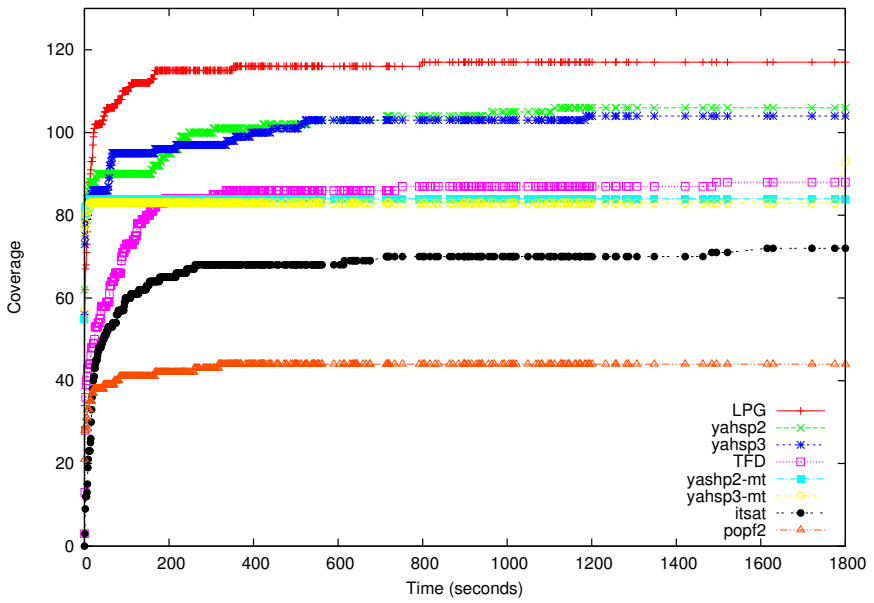

FIGURE 3. The number of solved instances over time of the considered planners on the benchmarks from the IPC 2014 temporal track. 


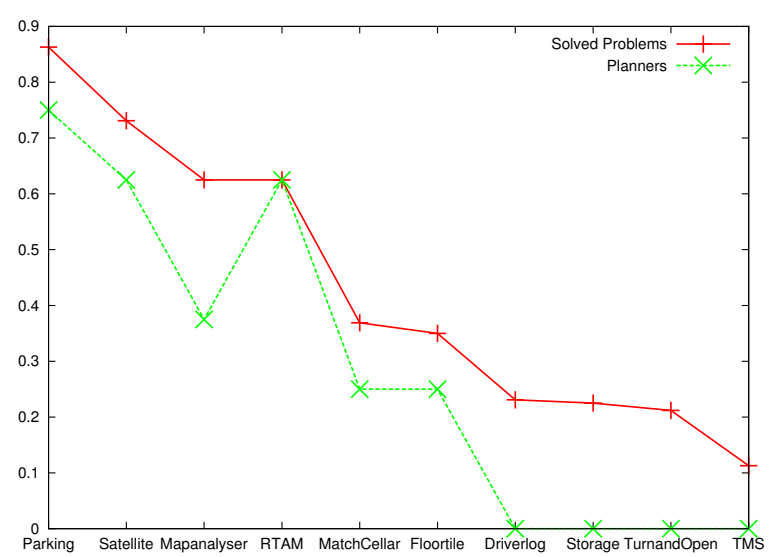

FIGURE 4. The red line (Solved Problems) is the proportion of the problems solved by all the planners. The green line (Planners) is the proportion of the planners that solved all the problems in the particular domain. 


\begin{tabular}{l|cl}
\hline Name & Type & Description \\
\hline Requirements & Integer & $\begin{array}{l}\text { Number of PDDL features that are in- } \\
\text { cluded in the domain definition. } \\
\text { Objects }\end{array}$ \\
Integer & $\begin{array}{l}\text { Number of types in the domain definition. } \\
\text { Integer }\end{array}$ & $\begin{array}{l}\text { Number of declared objects in the problem } \\
\text { definition. }\end{array}$ \\
Eacts & Integer & $\begin{array}{l}\text { Number of predicates in the domain defini- } \\
\text { tion. }\end{array}$ \\
Non-Durative Actions & Integer & $\begin{array}{l}\text { Number of predicates included in the ini- } \\
\text { tial state of the problem definition. } \\
\text { Number of non-durative actions included } \\
\text { in the domain definition. }\end{array}$ \\
Axioms & Integer & $\begin{array}{l}\text { Number of axioms included in the domain } \\
\text { definition }\end{array}$ \\
\hline \hline
\end{tabular}

TABLE 1. Propositional PDDL Features 


\begin{tabular}{|c|c|c|}
\hline Name & Type & Description \\
\hline Assignment & Integer & $\begin{array}{l}\text { Number of numeric assignments in the prob- } \\
\text { lem. }\end{array}$ \\
\hline Num durative actions & Integer & $\begin{array}{l}\text { Number of durative actions included in the } \\
\text { domain definition. }\end{array}$ \\
\hline numeric duration & Integer & $\begin{array}{l}\text { Number of durative actions with numeric du- } \\
\text { ration. }\end{array}$ \\
\hline function duration & Integer & $\begin{array}{l}\text { Number of durative actions with a numeric } \\
\text { fluent representing the duration. }\end{array}$ \\
\hline Avg numeric duration & Double & $\begin{array}{l}\text { Average, minimum and maximum duration } \\
\text { of durative actions with numeric duration. }\end{array}$ \\
\hline Functions & Double & $\begin{array}{l}\text { Number of numeric fluents included in the } \\
\text { domain definition }\end{array}$ \\
\hline Avg arity & Double & $\begin{array}{l}\text { Average, minimum and maximum of the ar- } \\
\text { ity of numeric fluents included in the do- } \\
\text { main. }\end{array}$ \\
\hline At_start condition & Double & $\begin{array}{l}\text { Average, minimum, maximum and standard } \\
\text { deviation of "at start" conditions. }\end{array}$ \\
\hline Over_all condition & Double & $\begin{array}{l}\text { Average, minimum, maximum and standard } \\
\text { deviation of "over all" conditions. }\end{array}$ \\
\hline At_end condition & Double & $\begin{array}{l}\text { Average, minimum, maximum and standard } \\
\text { deviation of "at end" conditions. }\end{array}$ \\
\hline At_start effect & Double & $\begin{array}{l}\text { Average, minimum, maximum and standard } \\
\text { deviation of "at start" effects. }\end{array}$ \\
\hline At_end effect & Double & $\begin{array}{l}\text { Average, minimum, maximum and standard } \\
\text { deviation of "at end" effects. }\end{array}$ \\
\hline
\end{tabular}

TABLE 2. Temporal PDDL Features 


\begin{tabular}{|c|c|c|}
\hline Name & Type & Description \\
\hline Num_VariablesCG & Integer & Number of Variables in the $\mathrm{CG}$ \\
\hline high Level VariablesCG & Integer & $\begin{array}{l}\text { Number of Variables that has at least one } \\
\text { goal. }\end{array}$ \\
\hline total EdgesCG & Integer & $\begin{array}{l}\text { Number of edges that connect the nodes in } \\
\text { the } C G\end{array}$ \\
\hline total WeightCG & Integer & Sum of the weight of the edges in the CG. \\
\hline veRatio & Double & Ratio between variables and edges in the CG. \\
\hline weRatio & Double & Ratio between weight and edges in the CG. \\
\hline wvRatio & Double & $\begin{array}{l}\text { Ratio between weight and variables in the } \\
\text { CG. }\end{array}$ \\
\hline hvRatio & Double & $\begin{array}{l}\text { Ratio between high level variables and the } \\
\text { other varaibles. }\end{array}$ \\
\hline input Edge & Double & $\begin{array}{l}\text { Maximum, average, standard deviation of the } \\
\text { input edges at the CG. }\end{array}$ \\
\hline output Edge & Double & $\begin{array}{l}\text { Maximum, average, standard deviation of the } \\
\text { output edges at the CG. }\end{array}$ \\
\hline input Weight & Double & $\begin{array}{l}\text { Maximum, average, standard deviation of the } \\
\text { weight of the input edges at the CG. }\end{array}$ \\
\hline output Weight & Double & $\begin{array}{l}\text { Maximum, average, standard deviation of the } \\
\text { weight of the output edges at the CG. }\end{array}$ \\
\hline input EdgeHV & Double & $\begin{array}{l}\text { Maximum, average, standard deviation of the } \\
\text { input edges at the High Level. }\end{array}$ \\
\hline output EdgeHV & Double & $\begin{array}{l}\text { Maximum, average, standard deviation of the } \\
\text { output edges at the High Level. }\end{array}$ \\
\hline input WeightHV & Double & $\begin{array}{l}\text { Maximum, average, standard deviation of the } \\
\text { weight of input edges at the High Level. }\end{array}$ \\
\hline output WeightHV & Double & $\begin{array}{l}\text { Maximum, average, standard deviation of the } \\
\text { weight of output edges at the High Level. }\end{array}$ \\
\hline
\end{tabular}

TABLE 3. General SAS+ Features extracted by considering the Causal Graph. 


\begin{tabular}{|c|c|c|}
\hline Name & Type & Description \\
\hline total Edges & Double & Number of edges of all DTGs. \\
\hline total Weigth & Double & Total weight of the edges of all DTGs. \\
\hline edVa Ratio DTG & Double & Ratio between edges and variables. \\
\hline weEd Ratio DTG & Double & Ratio between weight and edges. \\
\hline weVa Ratio DTG & Double & Ratio between weight and variables. \\
\hline input Edge DTG & Double & $\begin{array}{l}\text { Maximum, average, standard deviation of the input edges } \\
\text { of the DTG. }\end{array}$ \\
\hline output Edge & Double & $\begin{array}{l}\text { Maximum, average, standard deviation of the output edges } \\
\text { of the DTG. }\end{array}$ \\
\hline input Weight & Double & $\begin{array}{l}\text { Maximum, average, standard deviation of the weight of the } \\
\text { input edges of the DTG. }\end{array}$ \\
\hline output Weight & Double & $\begin{array}{l}\text { Maximum, average, standard deviation of the weight of the } \\
\text { output edges of the DTG. }\end{array}$ \\
\hline
\end{tabular}

TABLE 4. General SAS+ Features derived from DTGs. 


\begin{tabular}{|c|c|c|}
\hline Name & Type & Description \\
\hline Durative actions & Numeric & $\begin{array}{l}\text { Number of durative actions identified by } \\
\text { TFD. }\end{array}$ \\
\hline Action counter & Numeric & $\begin{array}{l}\text { Number of different actions from the SAS+ } \\
\text { translation }\end{array}$ \\
\hline Function symbols & Numeric & Number of symbols identified by TFD. \\
\hline Generated rules & Numeric & $\begin{array}{l}\text { Number of rules generated by TFD in the } \\
\text { translation process. }\end{array}$ \\
\hline Final queue & Numeric & $\begin{array}{l}\text { Number of the elements that appear in the } \\
\text { planning queue. }\end{array}$ \\
\hline Translator variables & Numeric & $\begin{array}{l}\text { Number of temporal variables identified by } \\
\text { TFD. }\end{array}$ \\
\hline Translator derived variables & Numeric & $\begin{array}{l}\text { Number of temporal derived variables iden- } \\
\text { tified by TFD. }\end{array}$ \\
\hline Translator facts & Numeric & Number of temporal facts identified by TFD. \\
\hline Mutex key & Numeric & Number of mutexes \\
\hline Strips to sas & Numeric & $\begin{array}{l}\text { Number of auxiliary variables used in a tem- } \\
\text { poral SAS+ encoding }\end{array}$ \\
\hline Ranges & Numeric & $\begin{array}{l}\text { Number of different numeric variables with } \\
\text { different ranges. }\end{array}$ \\
\hline Goal list & Numeric & $\begin{array}{l}\text { Number of elements in the goal state of the } \\
\text { temporal task. }\end{array}$ \\
\hline Task init & Numeric & $\begin{array}{l}\text { Number of elements in the initial state of the } \\
\text { temporal task. }\end{array}$ \\
\hline Translator durative act & Numeric & Number of actions in the preprocess phase. \\
\hline Translator axiom & Numeric & Number of axioms in the translation phase. \\
\hline Translator num axioms & Numeric & $\begin{array}{l}\text { Number of simplified axioms in the transla- } \\
\text { tion phase. }\end{array}$ \\
\hline Translator num axioms by layer & Numeric & Number of actions per level \\
\hline Translator max num layer & Numeric & Maximum number of layers \\
\hline
\end{tabular}

TABLE 5. Temporal SAS+ Features part I 


\begin{tabular}{|c|c|c|}
\hline Name & Type & Description \\
\hline Translator num axiom map & Numeric & $\begin{array}{l}\text { Number of axioms that appear throughout the } \\
\text { process }\end{array}$ \\
\hline Translator const num axioms & Numeric & Minimum number of necessary axioms \\
\hline Translator reachable & Numeric & $\begin{array}{l}\text { Number of variables that are reachable in the } \\
\text { initial state. }\end{array}$ \\
\hline Translator mutex group & Numeric & Number of mutex groups. \\
\hline Translation key & Numeric & Auxiliary value of TFD. \\
\hline Avg level & Numeric & Average number of levels. \\
\hline Std level & Numeric & Standard deviation of the number of levels. \\
\hline Global num type start & Numeric & $\begin{array}{l}\text { Number of transitions that are labeled at } \\
\text { at_start. }\end{array}$ \\
\hline Global num type end & Numeric & $\begin{array}{l}\text { Number of transitions that are labeled at } \\
\text { at_end. }\end{array}$ \\
\hline Global min level & Numeric & Minimum number of levels in DTGs. \\
\hline Global max level & Numeric & Maximum number of levels in DTGs. \\
\hline Global total level & Numeric & Total number of levels in DTGs. \\
\hline Init & Integer & $\begin{array}{l}\text { Number of predicates that appear in the initial } \\
\text { state. }\end{array}$ \\
\hline Goals & Integer & Number of predicates that appear in the goal. \\
\hline Function administrator & Integer & Auxiliary number of functions in TFD \\
\hline Final queue length & Integer & Size of the queue in the translation process. \\
\hline Translator operators & Integer & $\begin{array}{l}\text { Number of operators that appear in the trans- } \\
\text { lation process. }\end{array}$ \\
\hline Necessary operators & Integer & $\begin{array}{l}\text { Number of operators at the preprocessing } \\
\text { phase. }\end{array}$ \\
\hline Uncovered facts & Integer & $\begin{array}{l}\text { Number of facts included in the preprocess- } \\
\text { ing phase. }\end{array}$ \\
\hline Necessary variables & Integer & $\begin{array}{l}\text { Number of variables that appear in the trans- } \\
\text { lation process. }\end{array}$ \\
\hline Relation axioms & Integer & Number of axioms that are relational in TFD. \\
\hline Functional axioms & Integer & $\begin{array}{l}\text { Number of axioms that are functional in } \\
\text { TFD. }\end{array}$ \\
\hline True axioms & Integer & $\begin{array}{l}\text { Number of axioms that are true in the trans- } \\
\text { lation process. }\end{array}$ \\
\hline
\end{tabular}

TABLE 6. Temporal SAS+ Features part II 


\begin{tabular}{|c|c|c|}
\hline Name & Type & Description \\
\hline Ratio relevant actions & Double & $\begin{array}{l}\text { Ratio between the number of final and initial } \\
\text { actions. }\end{array}$ \\
\hline Num action & Integer & Number of final actions. \\
\hline Num propositions & Integer & Number of all propositions. \\
\hline Num relevant actions & Integer & Number of the final instantiated actions. \\
\hline Num relevant propositions & Integer & $\begin{array}{l}\text { Number of propositions that are included in } \\
\text { the relevant actions. }\end{array}$ \\
\hline Variables end & Integer & Created Variables in the SAT formulation. \\
\hline Propositions end & Double & $\begin{array}{l}\text { Number of proposition that are included in } \\
\text { the instantiated actions. }\end{array}$ \\
\hline Actions end & Integer & $\begin{array}{l}\text { Instantiated actions in the SAT formulation } \\
\text { after simplification. }\end{array}$ \\
\hline Total Mutex clauses & Double & Number of mutex clauses. \\
\hline Ratio end & Integer & $\begin{array}{l}\text { Ratio of the number of variables to the num- } \\
\text { ber of clauses }\end{array}$ \\
\hline Event clauses & Double & Number of clauses in the original formula. \\
\hline TClauses & Integer & Number of simplification clauses. \\
\hline Number Files & Integer & Number of temporal files needed by ITSAT. \\
\hline
\end{tabular}

TABLE 7. SAT Size Features extracted by considering the SAT-based encoding exploited by ITSAT. 


\begin{tabular}{ll|rr|rr}
\hline & & Average & Maximum & $\#$ & Succ. \\
\hline PDDL & Prop & 0.01 & 0.15 & 8 & $100 \%$ \\
& Temp & 5.06 & 10.00 & 28 & $100 \%$ \\
\hline SAT size & & 0.89 & 2.00 & 13 & $80 \%$ \\
\hline SAS+ & 28.89 & 50.00 & 90 & $80 \%$ \\
\hline \hline \multicolumn{2}{c|}{ Total } & 33.96 & 60.15 & 139 & - \\
\hline
\end{tabular}

TABLE 8. Average and Maximum CPU time needed to extract features, the number of features per group (\#) and the percentage of successful feature extraction (Succ.). 


\begin{tabular}{|l|l|}
\hline \multicolumn{2}{|c|}{ Training domains } \\
\hline IPC-2008 & IPC-2011 \\
\hline Crewplanning & Crewplanning \\
Elevators-N & Elevators \\
Elevators & Floortile \\
Modeltrain & Matchcellar \\
Openstacks-adl & Openstacks \\
Openstacks-N & Parcprinter \\
Openstacks-NADL & Parking \\
Openstacks & \\
Parcprinter & Pegsol \\
Pegsol & Sokoban \\
Sokoban & Storage \\
Transport & Temporal Machine Shop \\
Woodworking & Turn and Open \\
\hline
\end{tabular}

TABLE 9. Training domains categorised according to the planning competition in which they were used. 


\begin{tabular}{|c|c|c|c|c|c|c|c|c|}
\hline \# & IPC & Domain & IPC 2014 & Unknown & Known & ADL & Numeric & Durative-actions \\
\hline 1 & 2002 & Depots-simple-T & & $\checkmark$ & & & & $\checkmark$ \\
\hline 2 & & Depots-T & & $\checkmark$ & & & & $\checkmark$ \\
\hline 3 & & DriverLog-time & & $\checkmark$ & & & $\checkmark$ & $\checkmark$ \\
\hline 4 & & DriverLog-simpleTime & & $\checkmark$ & & & & $\checkmark$ \\
\hline 5 & & ZenoTravel-simpleTime & & $\checkmark$ & & & & $\checkmark$ \\
\hline 6 & & ZenoTravel-time & & $\checkmark$ & & & $\checkmark$ & $\checkmark$ \\
\hline 7 & & satellite & & $\checkmark$ & & & & $\checkmark$ \\
\hline 8 & & Rovers-mt & & $\checkmark$ & & & $\checkmark$ & \\
\hline 9 & & Rovers-time & & $\checkmark$ & & & $\checkmark$ & $\checkmark$ \\
\hline 10 & & UMLS-flaw & & $\checkmark$ & & & $\checkmark$ & $\checkmark$ \\
\hline 11 & & UMLS-fluents & & $\checkmark$ & & & $\checkmark$ & $\checkmark$ \\
\hline 12 & 2004 & Airport-adl & & $\checkmark$ & & $\checkmark$ & & $\checkmark$ \\
\hline 13 & & Airport-str & & $\checkmark$ & & & & $\checkmark$ \\
\hline 14 & & Pipesworld-mt & & $\checkmark$ & & & $\checkmark$ & \\
\hline 15 & & Pipesworld-mtc & & $\checkmark$ & & & $\checkmark$ & $\checkmark$ \\
\hline 17 & & Satellite-adl & & $\checkmark$ & & $\checkmark$ & & \\
\hline 18 & & NOTANKAGE & & $\checkmark$ & & & $\checkmark$ & $\checkmark$ \\
\hline 19 & & TANKAGE & & $\checkmark$ & & & $\checkmark$ & $\checkmark$ \\
\hline 20 & 2006 & Temporal Machine Shop (TMS) & & & $\checkmark$ & & & \\
\hline 21 & & Openstacks-strips & & & $\checkmark$ & $\checkmark$ & & \\
\hline 22 & & Openstacks-time & & & $\checkmark$ & $\checkmark$ & $\checkmark$ & $\checkmark$ \\
\hline 23 & & Openstacks-mt & & & $\checkmark$ & & $\checkmark$ & \\
\hline 24 & & Openstacks & & & $\checkmark$ & & $\checkmark$ & $\checkmark$ \\
\hline 25 & & Storage-time & & & $\checkmark$ & & & $\checkmark$ \\
\hline 26 & & Storage & & & $\checkmark$ & & & \\
\hline 27 & & Trucks-adl & & $\checkmark$ & $\checkmark$ & $\checkmark$ & $\checkmark$ & $\checkmark$ \\
\hline 28 & & Trucks-time & & $\checkmark$ & & & $\checkmark$ & \\
\hline 29 & & Rovers & & $\checkmark$ & & & $\checkmark$ & $\checkmark$ \\
\hline 30 & & PipesWorld & & $\checkmark$ & & & $\checkmark$ & $\checkmark$ \\
\hline 31 & 2014 & Driverlog & $\checkmark$ & & & & & $\checkmark$ \\
\hline 32 & & Floortile & $\checkmark$ & & & & & \\
\hline 33 & & Map-Analyser & $\checkmark$ & & & & & $\checkmark$ \\
\hline 34 & & Matchcellar & $\checkmark$ & & & & & $\checkmark$ \\
\hline 35 & & Parking & $\checkmark$ & & & & & $\checkmark$ \\
\hline 36 & & RTAM & $\checkmark$ & & & & & $\checkmark$ \\
\hline 37 & & Satellite & $\checkmark$ & & & & & $\checkmark$ \\
\hline 38 & & Storage & $\checkmark$ & & & & & $\checkmark$ \\
\hline 39 & & Temporal Machine Shop (TMS) & $\checkmark$ & & & & & $\checkmark$ \\
\hline 40 & & Turn and Open & $\checkmark$ & & & & & $\checkmark$ \\
\hline Total & & & $10(4 / 6)$ & 23 & 7 & & & \\
\hline
\end{tabular}

TABLE 10. The considered domains divided into the Unknown, Known and IPC 2014 sets. PDDL requirements per each considered domain. 


\begin{tabular}{|l|l|l|l|l|l|l|}
\hline Group & PDDL & SAS+ & Temporal (T) & Classical(nT) & Selection (Sel) & All \\
\hline Proposisitional PDDL & $\checkmark$ & & & $\checkmark$ & $\checkmark$ & 8 \\
Temporal PDDL & $\checkmark$ & & $\checkmark$ & & $\checkmark$ & 28 \\
General SAS+ & & $\checkmark$ & & $\checkmark$ & $\checkmark$ & 49 \\
Temporal SAS+ & & $\checkmark$ & $\checkmark$ & & & 30 \\
TFD & & & $\checkmark$ & & & 11 \\
SAT size & & & & 68 & 11 & 13 \\
\hline Total & 49 & 90 & 71 & 68 & 139 \\
\hline
\end{tabular}

TABLE 11. An overview of the sets of features considered in our experimental analysis. Checkmark indicates that the group of features (column) includes the corresponding set (row). The Sel set does not include all the features of involved groups. 


\begin{tabular}{l|llllll}
\hline \multirow{2}{*}{ Planner } & All & PDDL & SAS+ & nT & T & Sel \\
\hline LPG & 92.6 & 88.5 & 88.6 & $\mathbf{9 2 . 7}$ & 91.9 & 88.4 \\
POPF2 & 88.6 & 87.2 & 84.9 & $\mathbf{8 8 . 7}$ & 88.2 & 87.7 \\
Yahsp2 & 89.6 & 91.0 & 89.1 & 87.9 & 89.9 & $\mathbf{9 1 . 4}$ \\
Yahsp2-MT & $\mathbf{9 5 . 5}$ & 91.9 & 89.3 & 93.9 & 95.3 & 89.8 \\
ITSAT & $\mathbf{9 4 . 1}$ & 88.2 & 88.4 & 93.6 & 94.1 & 89.1 \\
TFD & 94.1 & 87.5 & 84.9 & 93.5 & $\mathbf{9 4 . 2}$ & 88.8 \\
Yahsp3 & 91.0 & 90.8 & 89.0 & 89.7 & 91.2 & $\mathbf{9 3 . 1}$ \\
Yahsp3-MT & $\mathbf{9 3 . 9}$ & 93.4 & 90.7 & 92.2 & 93.8 & 90.7 \\
\hline \hline
\end{tabular}

TABLE 12. Accuracy (higher is better) of the classification EPMs predicting whether a planner will solve a problem or not on the training instances. Bold indicates the best results (also considering hidden decimals). 


\begin{tabular}{|c|c|c|c|c|c|c|}
\hline \multicolumn{7}{|c|}{ IPC 2014} \\
\hline Planner & All & PDDL & SAS+ & nT & $\mathrm{T}$ & Sel \\
\hline LPG & 76.5 & 81.5 & 73.0 & 75.0 & 74.5 & 76.0 \\
\hline POPF2 & 87.0 & 77.5 & 83.5 & 86.5 & 80.5 & 68.5 \\
\hline Yahsp2 & 74.5 & 76.0 & 67.5 & 57.0 & 59.5 & 56.5 \\
\hline Yahsp2-MT & 63.5 & 80.5 & 65.0 & 72.5 & 57.0 & 68.0 \\
\hline ITSAT & 89.0 & 88.5 & 73.0 & 84.5 & 88.5 & 74.5 \\
\hline TFD & 67.0 & 67.0 & 69.5 & 71.0 & 67.0 & 67.0 \\
\hline Yahsp3 & 60.0 & 74.0 & 61.5 & 59.0 & 57.0 & 56.0 \\
\hline Yahsp3-MT & 75.0 & 82.0 & 73.0 & 65.5 & 57.0 & 78.0 \\
\hline \multicolumn{7}{|c|}{ Known } \\
\hline & All & PDDL & SAS+ & $\mathrm{nT}$ & $\mathrm{T}$ & Sel \\
\hline LPG & 42.5 & 81.2 & 33.3 & 31.2 & 76.3 & 53.8 \\
\hline POPF2 & 65.6 & 72.0 & 67.20 & 45.7 & 77.4 & 51.6 \\
\hline Yahsp2 & 43.6 & 75.8 & 74.19 & 76.9 & 78.0 & 78.0 \\
\hline Yahsp2-MT & 80.1 & 57.5 & 76.9 & 76.3 & 79.0 & 79.0 \\
\hline ITSAT & 97.3 & 100 & 76.3 & 86.0 & 98.4 & 92.5 \\
\hline TFD & 42.5 & 39.3 & 71.5 & 75.3 & 44.6 & 41.4 \\
\hline Yahsp3 & 71.5 & 77.4 & 65.1 & 74.7 & 57.5 & 77.4 \\
\hline Yahsp3-MT & 53.2 & 78.5 & 76.9 & 75.8 & 78.5 & 78.5 \\
\hline \multicolumn{7}{|c|}{ Unknown } \\
\hline & All & PDDL & SAS+ & $\mathrm{nT}$ & $\mathrm{T}$ & Sel \\
\hline LPG & 62.6 & 48.9 & 64.4 & 55.4 & 55.7 & 56.8 \\
\hline POPF2 & 59.8 & 57.0 & 67.3 & 77.1 & 42.1 & 57.1 \\
\hline Yahsp2 & 48.9 & 80.3 & 84.7 & 84.5 & 73.2 & 75.7 \\
\hline Yahsp2-MT & 75.0 & 71.4 & 79.4 & 82.2 & 74.7 & 72.0 \\
\hline ITSAT & 92.4 & 86.2 & 90.0 & 75.0 & 89.3 & 92.4 \\
\hline TFD & 57.6 & 53.7 & 70.8 & 68.0 & 40.8 & 30.6 \\
\hline Yahsp3 & 62.2 & 73.7 & 78.2 & 71.9 & 78.2 & 55.7 \\
\hline Yahsp3-MT & 86.4 & 65.0 & 78.3 & 72.7 & 73.6 & 57.1 \\
\hline
\end{tabular}

TABLE 13. Accuracy (higher is better) of the classification EPMs predicting whether a planner will solve a problem or not on the testing instances. Bold indicates the best results (also considering hidden decimals). 


\begin{tabular}{l|llllll}
\hline \multirow{2}{*}{ Planner } & All & PDDL & SAS+ & nT & T & Sel \\
\hline LPG & 1.49 & 1.57 & 1.84 & 1.54 & $\mathbf{1 . 4 8}$ & 1.49 \\
POPF2 & 2.12 & 2.27 & 2.53 & 2.23 & 2.11 & $\mathbf{2 . 0 5}$ \\
Yahsp2 & 1.76 & 1.45 & 2.07 & 1.86 & 1.65 & $\mathbf{1 . 2 7}$ \\
Yahsp2-MT & 1.41 & 1.45 & 2.25 & 1.84 & 1.33 & $\mathbf{1 . 3 0}$ \\
ITSAT & 1.45 & 1.58 & 1.68 & 1.41 & 1.42 & $\mathbf{1 . 3 8}$ \\
TFD & 2.18 & 2.32 & 2.56 & 2.19 & 2.16 & $\mathbf{2 . 0 2}$ \\
Yahsp3 & 1.61 & 1.60 & 2.04 & 1.81 & 1.43 & $\mathbf{1 . 4 1}$ \\
Yahsp3-MT & 1.42 & 1.28 & 1.29 & 1.55 & 1.21 & $\mathbf{1 . 1 7}$ \\
\hline \hline
\end{tabular}

TABLE 14. Root mean squared error (lower is better) of the regression EPMs built by using Decision Tables on training instances. Bold indicates the best performance (also considering hidden decimals). 


\begin{tabular}{|c|c|c|c|c|c|c|}
\hline \multicolumn{7}{|c|}{ IPC 2014} \\
\hline Planner & All & PDDL & SAS+ & nT & $\mathrm{T}$ & Sel \\
\hline LPG & 3.29 & 3.56 & 2.61 & 2.60 & 3.44 & 2.20 \\
\hline POPF2 & 2.49 & 2.43 & 2.22 & 2.84 & 2.48 & 2.76 \\
\hline Yahsp2 & 2.76 & 2.55 & 3.22 & 2.76 & 2.37 & 3.63 \\
\hline Yahsp2-MT & 2.83 & 3.05 & 3.36 & 3.08 & 2.89 & 2.86 \\
\hline ITSAT & 2.06 & 2.28 & 2.54 & 2.42 & 2.36 & 1.87 \\
\hline TFD & 2.51 & 2.73 & 2.87 & 2.80 & 2.83 & 2.19 \\
\hline Yahsp3 & 2.60 & 3.33 & 3.23 & 2.85 & 2.79 & 2.20 \\
\hline Yahsp3-MT & 2.99 & 2.85 & 3.12 & 3.27 & 2.65 & 2.64 \\
\hline \multicolumn{7}{|c|}{ Known } \\
\hline & All & PDDL & SAS+ & nT & $\mathrm{T}$ & Sel \\
\hline LPG & 3.02 & 3.02 & 3.54 & 3.53 & 3.02 & 3.02 \\
\hline POPF2 & 2.86 & 2.46 & 2.53 & 2.67 & 2.43 & 2.46 \\
\hline Yahsp2 & 1.73 & 1.57 & 2.03 & 2.06 & 1.57 & 1.62 \\
\hline Yahsp2-MT & 3.18 & 3.16 & 2.12 & 2.13 & 3.16 & 1.54 \\
\hline ITSAT & 1.61 & 1.61 & 0.87 & 0.99 & 2.15 & 2.29 \\
\hline TFD & 3.47 & 3.47 & 2.98 & 2.94 & 3.45 & 3.09 \\
\hline Yahsp3 & 1.46 & 1.51 & 2.12 & 2.12 & 1.47 & 1.57 \\
\hline Yahsp3-MT & 2.42 & 1.99 & 1.95 & 1.97 & 1.68 & 1.49 \\
\hline \multicolumn{7}{|c|}{ Unknown } \\
\hline & All & PDDL & SAS+ & $\mathrm{nT}$ & $\mathrm{T}$ & Sel \\
\hline LPG & 2.86 & 2.86 & 2.13 & 2.86 & 2.86 & 2.31 \\
\hline POPF2 & 2.26 & 2.35 & 2.15 & 2.06 & 2.35 & 2.36 \\
\hline Yahsp2 & 2.18 & 2.15 & 2.39 & 2.37 & 2.15 & 2.15 \\
\hline Yahsp2-MT & 2.80 & 2.78 & 2.40 & 2.35 & 2.78 & 2.02 \\
\hline ITSAT & 2.70 & 2.70 & 2.45 & 2.56 & 2.84 & 2.85 \\
\hline TFD & 3.86 & 3.86 & 2.81 & 2.78 & 3.86 & 2.80 \\
\hline Yahsp3 & 2.15 & 2.15 & 2.46 & 2.44 & 2.12 & 2.32 \\
\hline Yahsp3-MT & 2.13 & 2.03 & 2.16 & 2.20 & 2.02 & 1.88 \\
\hline
\end{tabular}

TABLE 15. Root mean squared error (lower is better) of regression EPMs built by using Decision Tables on testing instances. Bold indicates the best performance (also considering hidden decimals). 


\begin{tabular}{|c|c|c|c|c|c|c|c|c|c|c|c|c|}
\hline \multirow[b]{2}{*}{ Domain } & \multicolumn{4}{|c|}{ Classification } & \multicolumn{4}{|c|}{ Regression } & \multicolumn{2}{|c|}{ Best } & \multirow[t]{2}{*}{ VBS } & \multirow[t]{2}{*}{ B4P } \\
\hline & All & Sel & nT & $\mathrm{T}$ & All & Sel & $\mathrm{nT}$ & $\mathrm{T}$ & $\mathrm{C}$ & $S$ & & \\
\hline TMS & 18 & 18 & 16 & 18 & 18 & 18 & 18 & 18 & 0 & 0 & 18 & 0 \\
\hline TurnAndOpen & 12 & 12 & 14 & 15 & 17 & 17 & 17 & 17 & 0 & 0 & 17 & 15 \\
\hline Storage & 17 & 17 & 17 & 17 & 17 & 17 & 17 & 17 & 17 & 9 & 17 & 17 \\
\hline Driverlog & 7 & 2 & 6 & 0 & 13 & 0 & 13 & 13 & 13 & 9 & 13 & 12 \\
\hline Floortile & 20 & 20 & 20 & 20 & 20 & 20 & 20 & 20 & 20 & 8 & 20 & 20 \\
\hline MatchCellar & 19 & 20 & 20 & 20 & 20 & 20 & 20 & 20 & 0 & 0 & 20 & 20 \\
\hline MapAnalyser & 10 & 14 & 9 & 10 & 7 & 7 & 7 & 7 & 7 & 20 & 20 & 20 \\
\hline RTAM & 0 & 6 & 0 & 3 & 20 & 20 & 20 & 20 & 20 & 20 & 20 & 20 \\
\hline Satellite & 12 & 3 & 6 & 2 & 20 & 20 & 20 & 20 & 20 & 20 & 20 & 20 \\
\hline Parking & 14 & 20 & 20 & 20 & 20 & 20 & 20 & 20 & 20 & 20 & 20 & 20 \\
\hline Coverage & 129 & 132 & 128 & 125 & 172 & 159 & 172 & 172 & 117 & 106 & 185 & 164 \\
\hline IPC-Score & 91.8 & 102.4 & 95.1 & 105.8 & 129.3 & 126.6 & 129.3 & 129.3 & 62.1 & 86.2 & 185 & 72.5 \\
\hline
\end{tabular}

TABLE 16. Coverage and total IPC score of the regression and classification EPMs exploited for algorithm selection; of the best basic solver according to coverage (Best-C); of the best basic solver according to IPC score (S-Best), of the virtual best solver (VBS), and of a static portfolio including 4 planners (B4P). The rows in grey indicate the domains that are not included in the training set. Bold indicates the best performance. 


\begin{tabular}{|c|c|c|c|c|c|c|c|c|c|c|c|}
\hline \multicolumn{12}{|c|}{ Unknown Test Set } \\
\hline & \multicolumn{4}{|c|}{ Classification } & \multicolumn{4}{|c|}{ Regression } & \multirow[t]{2}{*}{ Best } & \multirow[t]{2}{*}{ VBS } & \multirow[t]{2}{*}{ B4P } \\
\hline & All & Sel & nT & $\mathrm{T}$ & All & Sel & nT & $\mathrm{T}$ & & & \\
\hline Total & 238 & 167 & 260 & 229 & 309 & 309 & 301 & 309 & 360 & 437 & 404 \\
\hline IPC-Score & 175.1 & 177.9 & 183.0 & 168.4 & 277.0 & 277.0 & 269.2 & 277.0 & 242.6 & 437 & 249.6 \\
\hline \multicolumn{12}{|c|}{ Known Test Set } \\
\hline & \multicolumn{4}{|c|}{ Classification } & \multicolumn{4}{|c|}{ Regression } & Best & VBS & B4P \\
\hline & All & Sel & nT & $\mathrm{T}$ & All & Sel & nT & $\mathrm{T}$ & & & \\
\hline Coverage & 79 & 78 & 71 & 78 & 115 & 111 & 115 & 114 & 143 & 162 & 153 \\
\hline IPC-Score & 50.0 & 57.3 & 64.2 & 54.8 & 113.1 & 110.2 & 110.2 & 108.6 & 88.7 & 162.0 & 90.0 \\
\hline
\end{tabular}

TABLE 17. Coverage and total IPC score of the regression and classification EPMs exploited for algorithm selection; of the best basic solver according to coverage (Best); of the virtual best solver (VBS), and of a static portfolio configured on the testing problems (B4P). Bold indicates the best performance. 


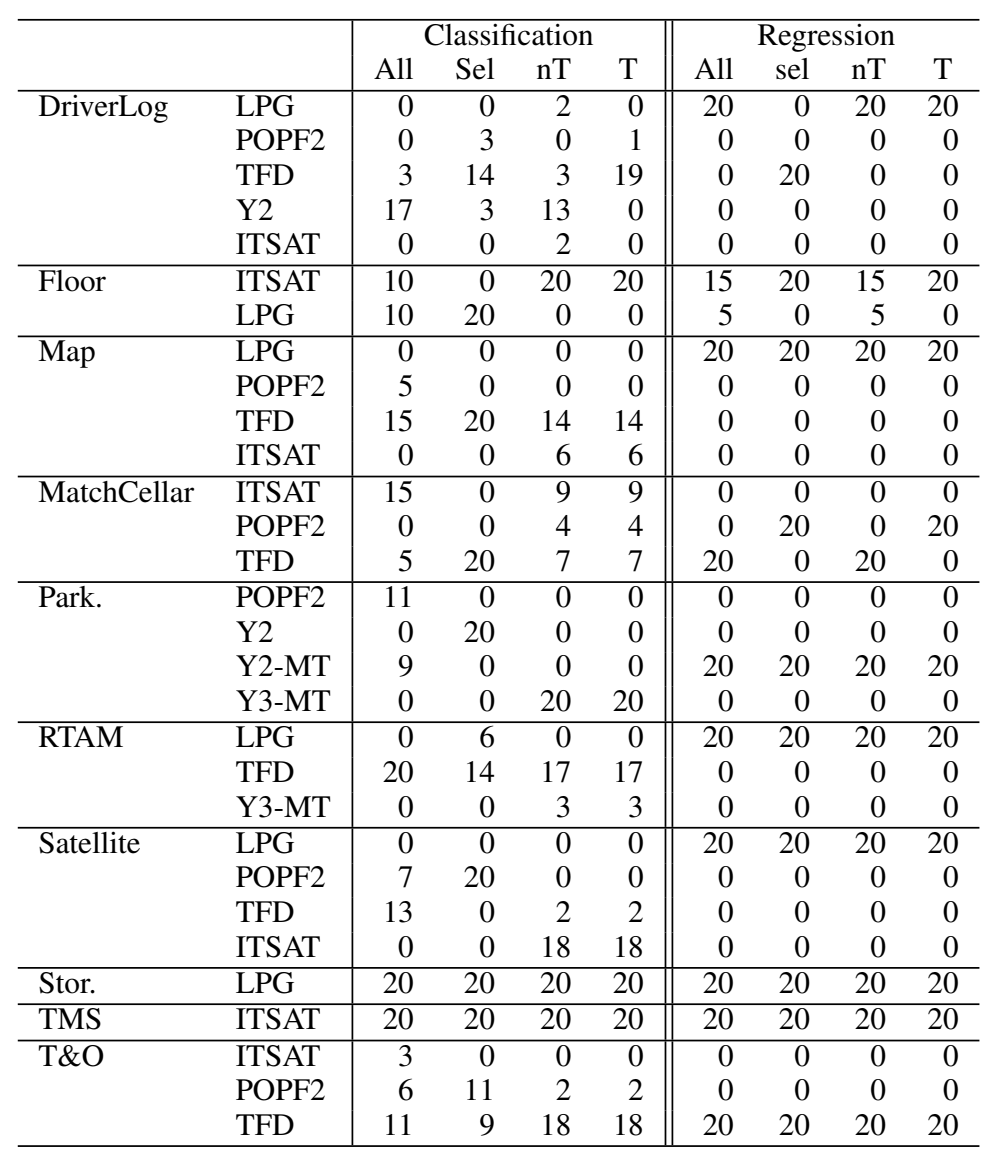

TABLE 18. Planners selected by the Classification or Regression EPMs, with different sets of features on the IPC 2014 benchamrks. 


\begin{tabular}{l|rrrr|rrrr} 
& \multicolumn{4}{|c|}{ Classification } & \multicolumn{4}{c}{ Regression } \\
& All & Sel & nT & T & All & Sel & nT & \multicolumn{1}{c}{ T } \\
\hline LPG & 30 & 46 & 22 & 20 & 105 & 80 & 100 & 105 \\
Yahsp2 & 17 & 23 & 13 & 0 & 0 & 0 & 0 & 0 \\
Yahsp2-MT & 9 & 0 & 0 & 0 & 20 & 20 & 20 & 20 \\
POPF2 & 29 & 34 & 6 & 7 & 0 & 20 & 20 & 0 \\
ITSAT & 48 & 20 & 57 & 55 & 35 & 40 & 40 & 35 \\
TFD & 67 & 77 & 61 & 77 & 40 & 40 & 20 & 40 \\
Yahsp3 & 0 & 0 & 0 & 0 & 0 & 0 & 0 & 0 \\
Yashp3-MT & 0 & 0 & 23 & 23 & 0 & 0 & 0 & 0 \\
\hline
\end{tabular}

TABLE 19. Number of times each planner has been selected by the classification or regression EPMs exploiting different sets of features. nT and T refer to Non-Temporal and Temporal sets of features, respectively. 